XVI CONGRESO LATINOAMERICANO DE PATOLOGÍA DE LA CONSTRUCCÍN
XVIII DE CONTROL DE CALIDAD EN LA CONSTRUCCIÓN

\title{
Análise dinâmica da superestrutura de uma ponte: alvo de ataques criminosos em 2019, em Tabuleiro do Norte, Ceará
}

\author{
J.Beserra Filho ${ }^{1 *}$, E. Mesquita ${ }^{2}$ \\ 1 LAREB, Engenharia Civil, UFC, Campus Russas, Russas, CE. \\ 2 LAREB, Engenharia Civil, UFC, Campus Russas, Russas, CE. \\ *Autor correspondente: joao_moradanova@hotmail.com
}

\section{RESUMO}

Este trabalho se dedica na compreensão do comportamento de uma ponte, frente as ações dinâmicas, dada as possíveis variáveis que podem influenciar na sua estrutura. Para o estudo, a superestrutura da ponte sobre o rio Jaguaribe, mais conhecida como Ponte do Peixe Gordo que está localizada na cidade de Tabuleiro do Norte-CE. São apresentadas as análises dinâmicas, que mostram a caracterização do comportamento da ponte, picos dos deslocamentos totais para cada modo de vibração. Conclui-se que os objetivos propostos no trabalho foram alcançados sendo que os resultados obtidos foram significativos, pois foi possível identificar as regiões mais propicias a excitação dinâmica, possibilitando a identificação de mecanismos de danos, contribuindo para a avaliação da segurança estrutural da ponte.

Palavras-chave: ponte; comportamento estrutural; análise dinâmica. 
This work is dedicated to understanding the behavior of a bridge, facing dynamic actions, given the various possibilities that can influence the structure. In this study, the superstructure above the Jaguaribe River, better known as Ponte do Peixe Gordo which is in the municipality of Tabuleiro do Norte-CE. Dynamic analyzes are presented, which show the characterization of the bridge's behavior, peaks of the total displacements for each vibration mode. It is concluded that the objectives proposed in the work were achieved and the results obtained were significant, as it was possible to identify the regions most propitious to dynamic excitation, enabling the identification of damage mechanisms, contributing to the assessment of the structural safety of the bridge.

Keywords: bridge; structural behavior; dynamic analysis.

\section{RESUMEN}

Este trabajo está dedicado a comprender el comportamiento de una puente, frente a acciones dinámicas, dadas las diversas posibilidades que pueden influir en su estructura. Para el estudio, la superestructura sobre el río Jaguaribe, más conocido como Ponte do Peixe Gordo que se encuentra en el municipio de Tabuleiro do Norte-CE. Se presentan análisis dinámicos, que muestran la caracterización del comportamiento del puente, picos de dos desplazamientos totales para cada modo de vibración. Se concluye que los objetivos propuestos en el trabajo fueron alcanzados y los resultados obtenidos fueron significativos, ya que fue posible identificar las regiones más propicias para la excitación dinámica, lo que permitió identificar los mecanismos de daño, contribuyendo a la evaluación de la seguridad estructural de la puente.

Palabras llave: puente; comportamiento estructural; análisis dinámico.

\section{INTRODUÇÃO}


A análise estrutural tem por objetivo associar os sistemas físicos com ações externas, aplicando modelos calibrados com os materiais e suas propriedades, tornando possível caracterizar o seu comportamento.

A dinâmica das estruturas tem por objetivo a determinação de deslocamentos, velocidades e acelerações, todos os elementos constituintes de uma estrutura submetida a cargas dinâmicas. Subjaz a isso, é possível constatar, de maneira menos precisa, a dinamicidade das ações sobre as estruturas e, concomitante a essa afirmativa, deve ser observado as forças de inércia e suas respectivas acelerações a que cada um de seus componentes fica sujeito.

Nesse contexto, tem-se a Ponte do Peixe Gordo, esta que foi alvo de ataques criminosos, a análise dinâmica faz-se viável e necessária, com o intuito de verificar a segurança estrutural, como também dispor de dados, que possam embasar em possíveis reparos e intervenções em sua estrutura.

Deste modo, a elaboração deste trabalho, se baseia no comportamento dinâmico da superestrutura da ponte do Peixe Gordo, caracterizando a estrutura sob simulação dinâmica computacional, identificando as frequências naturais, os deslocamentos e as participações de massa.

\section{ESTUDO DE CASO}

\subsection{A Ponte do Peixe Gordo}

A ponte do Peixe Gordo, foi construída em 1948, passando por intervenções para propiciar melhorias, no ano de 2001 houve a necessidade do alargamento da ponte, visto o crescimento do tráfego. Tem como obstáculo transposto, o rio Jaguaribe, sua extensão total é de 412 metros, sua superestrutura tem extensão de 390 metros, é composta pelos seguintes elementos estruturais: as longarinas (com alargamento nas proximidades dos pilares), a laje do tabuleiro (pista de rolamento, com acréscimo do revestimento e passarela de passeio), as guarda-rodas e os guarda-corpo.

A ponte foi alvo de ataques criminosos, os quais não ocasionaram danos relevantes a estrutura. Porém com o intuito de se verificar a segurança estrutural da ponte, alguns estudos técnicos foram realizados, dentre os quais, análises computacionais, das quais deriva este trabalho.

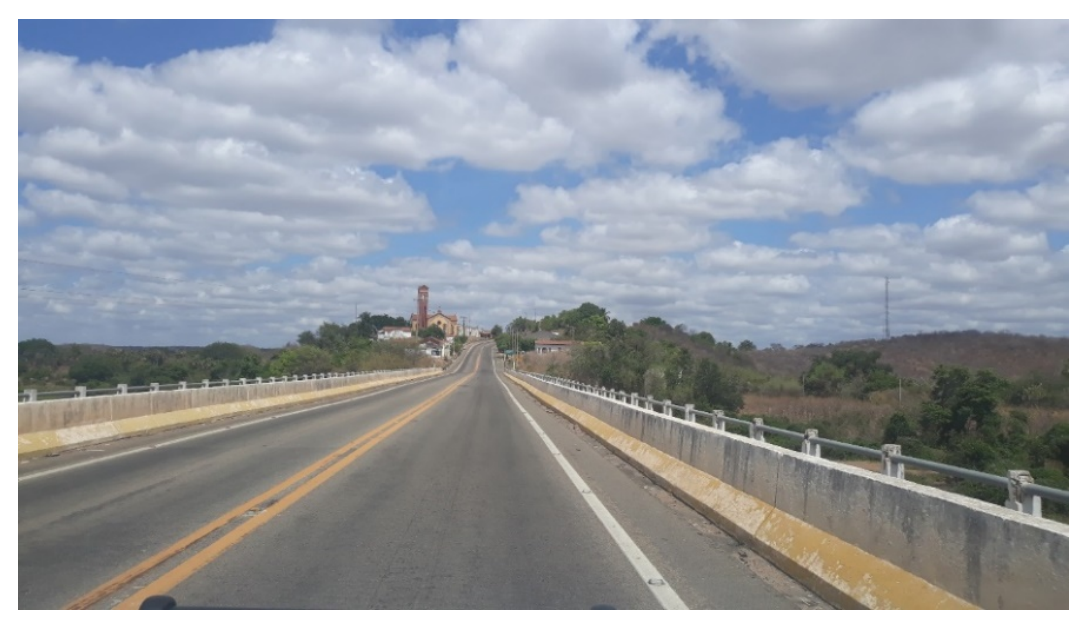

Figura 1. Ponte do Peixe Gordo. 


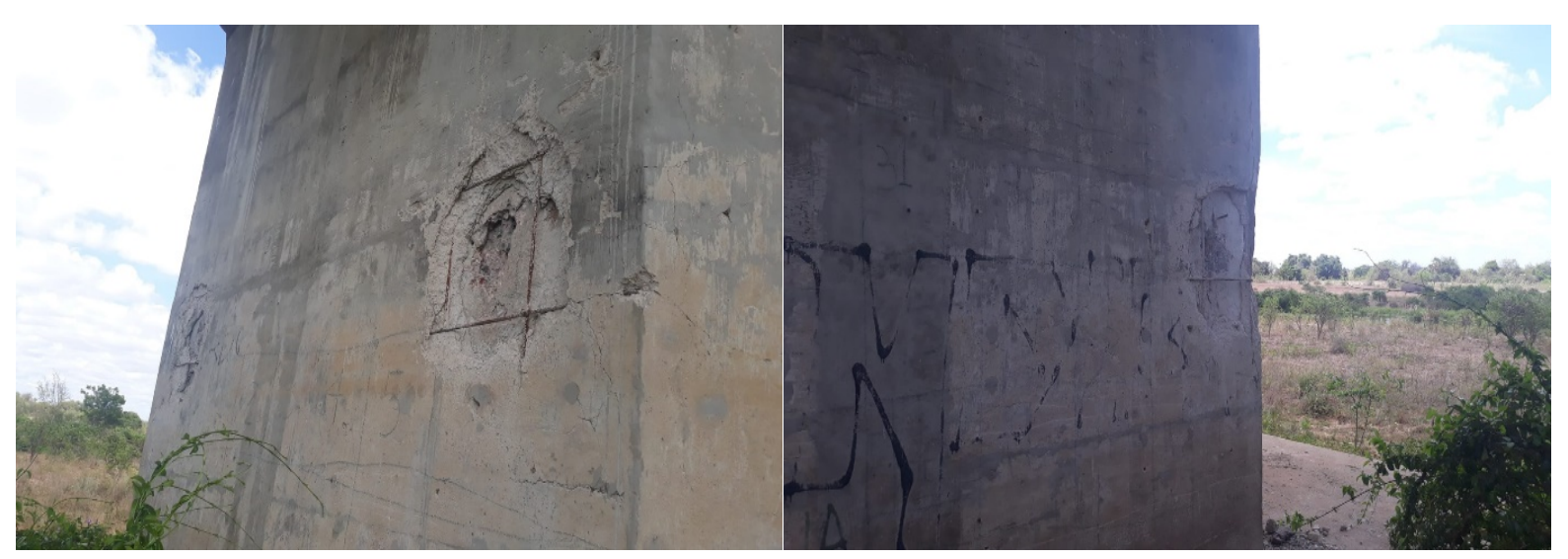

Figura 2. Danos causados por ataques criminosos em 2019.

\subsection{Modelagem numérica da estrutura}

Para a modelagem numérica, buscou-se a elaboração de um modelo 3D que representasse a superestrutura da ponte. Para isso foram utilizadas as informações de projetos do Departamento Nacional de Infraestrutura e Transportes (DNIT), onde possibilitou a construção do modelo que representasse a estrutura da ponte o mais fidedigno possível às suas características. Em seguida, o modelo foi importado para o no software Ansys ${ }^{\circledR}$, versão 17.1, para que se obtivesse a malha de elementos finitos. Com isso, obteve-se uma malha composta por 192.784 elementos e 394.252 nós. A Figura 3 mostra um vão do modelo 3D obtido.
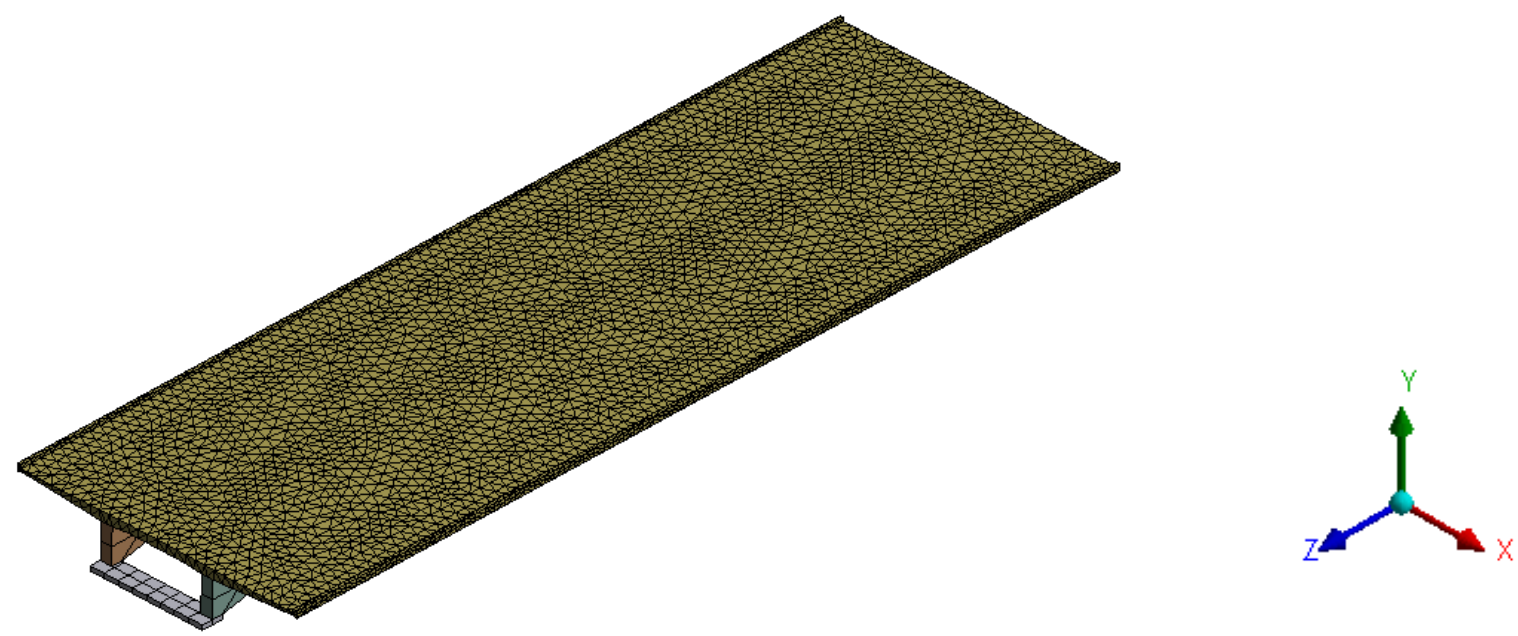

Figura 3. Modelo 3D em malha de elementos finitos.

\subsection{Carregamentos e propriedades mecânicas}

Os carregamentos aplicados nesta análise foram as ações permanentes das pontes, de acordo com a NBR 8681 (2003), são as cargas constantes, ou seja, peso próprio dos elementos da superestrutura. Os elementos da superestrutura que estão no modelo 3D são as vigas e a laje, para os demais elementos adotaram-se forças para substituir o peso próprio, são eles: guarda-rodas, guarda-corpo e revestimento, assim os carregamentos permanentes estão aplicados na estrutura, onde facilitou a construção da malha do modelo. 
Para o cálculo dos carregamentos, o material utilizado em todo o modelo foi o de características que se semelham ao concreto armado. Adotando assim, os seguintes parâmetros, encontrados em Pinheiro (2007), para as propriedades mecânicas do material: considerou-se o módulo de elasticidade $(\mathrm{E})$ de $30 \mathrm{GPa}$, o peso específico $(\mathrm{w})$ de $25 \mathrm{kN} / \mathrm{m}$, a resistência à compressão $\left(\mathrm{f}_{\mathrm{c}}\right) \mathrm{de}$ $25 \mathrm{MPa}$, a resistência a tração $\left(\mathrm{f}_{\mathrm{t}}\right)$ de $3 \mathrm{MPa}$ e o coeficiente de Poisson $(v)$ de 0,20 . As propriedades estão dispostas na Tabela 1.

Tabela 1. Propriedades mecânicas adotadas no modelo numérico para análise estática.

\begin{tabular}{|c|c|c|}
\hline Propriedade Mecânica & Magnitude & Unidade \\
\hline$E$ & 30,00 & $\mathrm{GPa}$ \\
\hline $\mathrm{W}$ & 25,00 & $\mathrm{kN} / \mathrm{m}^{3}$ \\
\hline $\mathrm{f}_{\mathrm{c}}$ & 25,00 & $\mathrm{MPa}$ \\
\hline $\mathrm{f}_{\mathrm{t}}$ & 3,00 & $\mathrm{MPa}$ \\
\hline$v$ & 0,20 & adimensional \\
\hline
\end{tabular}

\section{RESULTADOS}

\subsection{Frequências naturais e modos de vibração}

Foram extraídos as frequências naturais e períodos de 6 modos de vibração do modelo 3D. A Tabela 2 apresenta os resultados e as respectivas participações de massa para cada eixo, onde o eixo $\mathrm{X}$ corresponde ao sentido transversal horizontal da ponte, o eixo $\mathrm{Y}$ no sentido vertical e o eixo $\mathrm{Z}$ no sentido longitudinal horizontal.

Tabela 2. Frequência natural e período para cada modo de vibração.

\begin{tabular}{|c|c|c|c|c|c|}
\hline \multirow{2}{*}{$\begin{array}{l}\text { Modo de } \\
\text { Vibração }\end{array}$} & \multirow{2}{*}{$\begin{array}{l}\text { Frequência } \\
(\mathbf{H z})\end{array}$} & \multirow{2}{*}{$\begin{array}{c}\text { Período } \\
\text { (s) }\end{array}$} & \multicolumn{3}{|c|}{ Participação de Massa \% } \\
\hline & & & Eixo X & Eixo Y & Eixo Z \\
\hline 1 & 3,0902 & 0,324 & 7,64 & 1,64 & 0,00 \\
\hline 2 & 3,0994 & 0,323 & 2,46 & 0,00 & 5,24 \\
\hline 3 & 3,1154 & 0,321 & 21,47 & 17,07 & 0,00 \\
\hline 4 & 3,1383 & 0,319 & 5,99 & 0,00 & 22,95 \\
\hline 5 & 3,1690 & 0,316 & 50,26 & 81,29 & 0,01 \\
\hline 6 & 3,2094 & 0,312 & 12,17 & 0,00 & 71,79 \\
\hline
\end{tabular}

Com os dados extraídos, tem-se também as representações gráficas nos eixos $\mathrm{X}, \mathrm{Y}$ e $\mathrm{Z}$, onde destaca-se as participações de massa no eixo principal do gráfico, no eixo secundário temos as frequências naturais, onde se tem o acréscimo das frequências ao passo que as simulações se desenvolvem. 


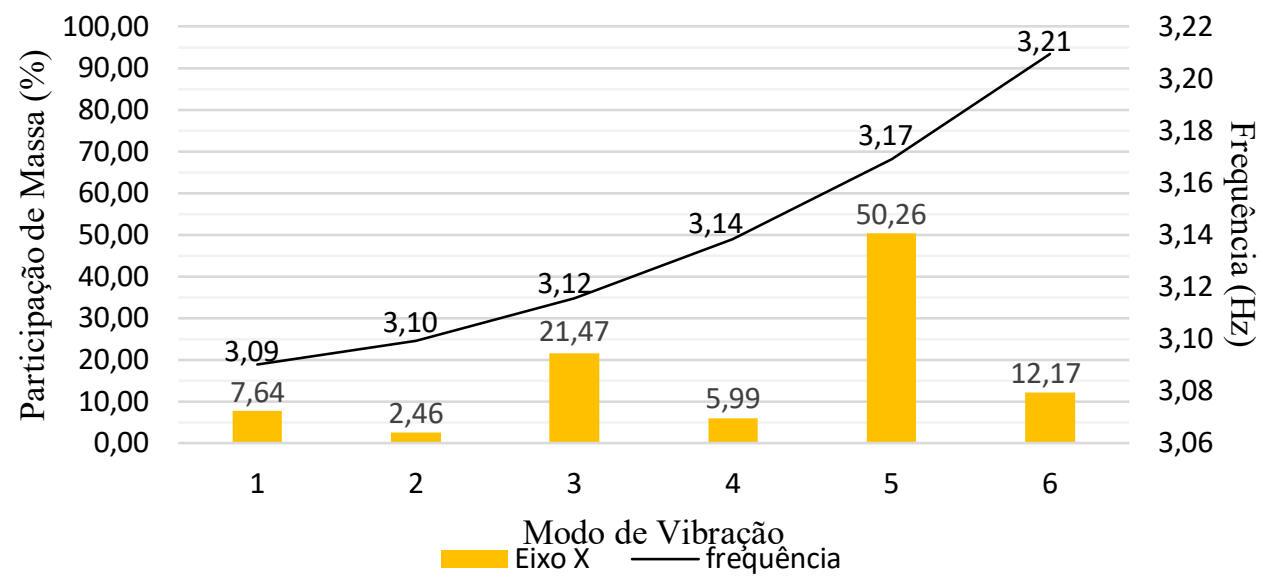

Figura 4. Representação gráfica da participação de massa no eixo X, e também, das frequências naturais de vibração.

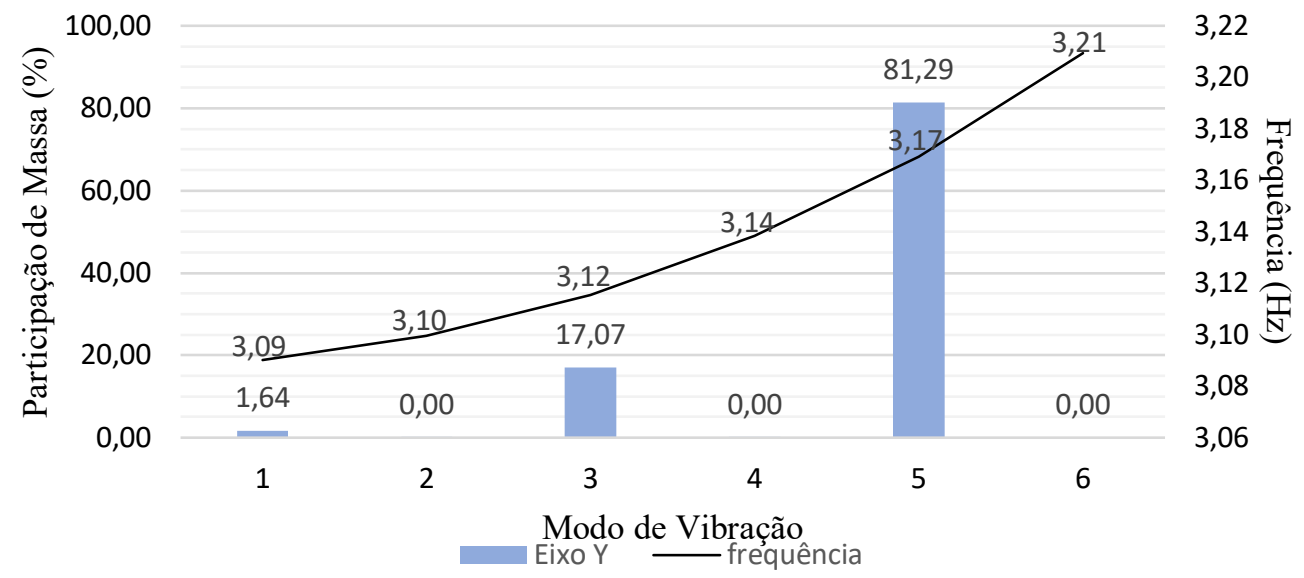

Figura 5. Representação gráfica da participação de massa no eixo Y, e também, das frequências naturais de vibração.

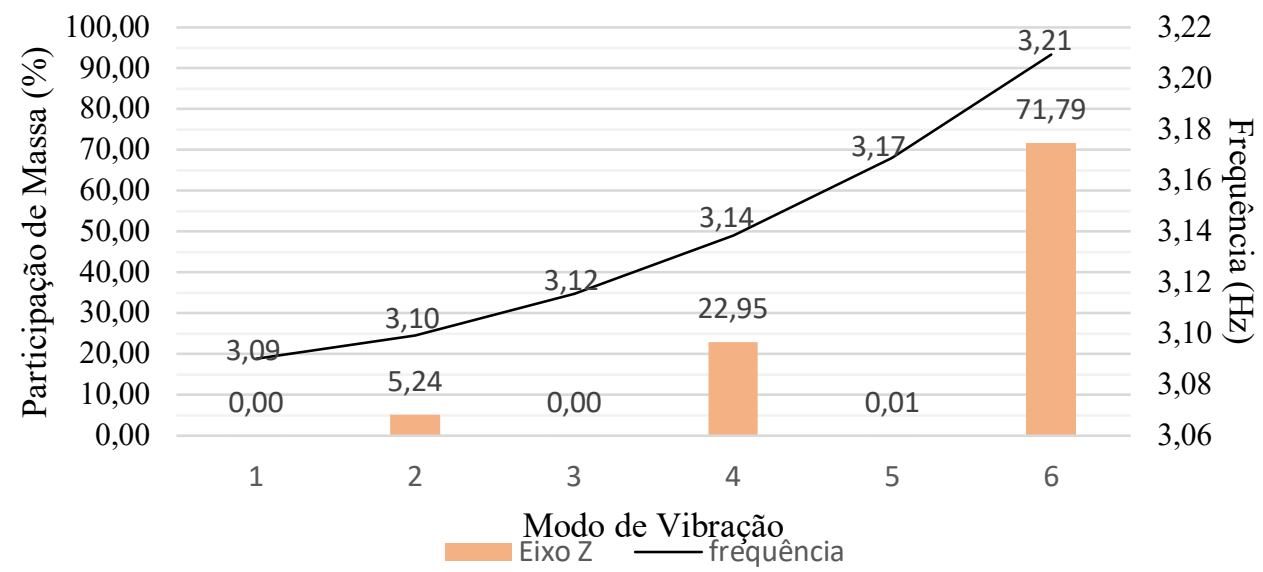

Figura 6. Representação gráfica da participação de massa no eixo Z, e também, das frequências naturais de vibração. 
O eixo Y, eixo vertical, foi o que apresentou deslocamentos significativos, em relação aos demais eixos, $\mathrm{X}$ e Z, pois a superestrutura da ponte está no plano $\mathrm{XZ}$, sendo assim os apoios fixados estão no mesmo plano, consequentemente as vibrações atuam de forma direta no eixo Y. Isso pode ser percebido nos 6 modos de vibração obtidos, e apresentados a seguir.
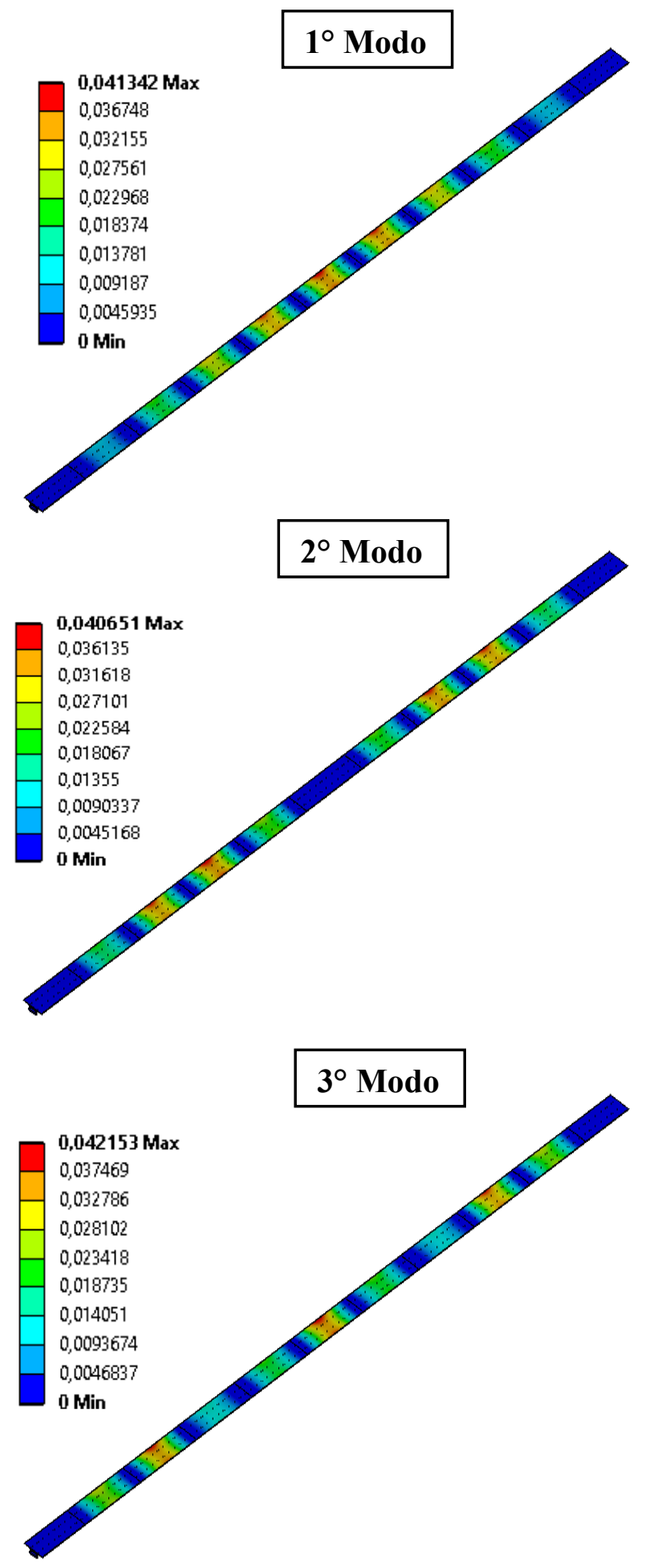
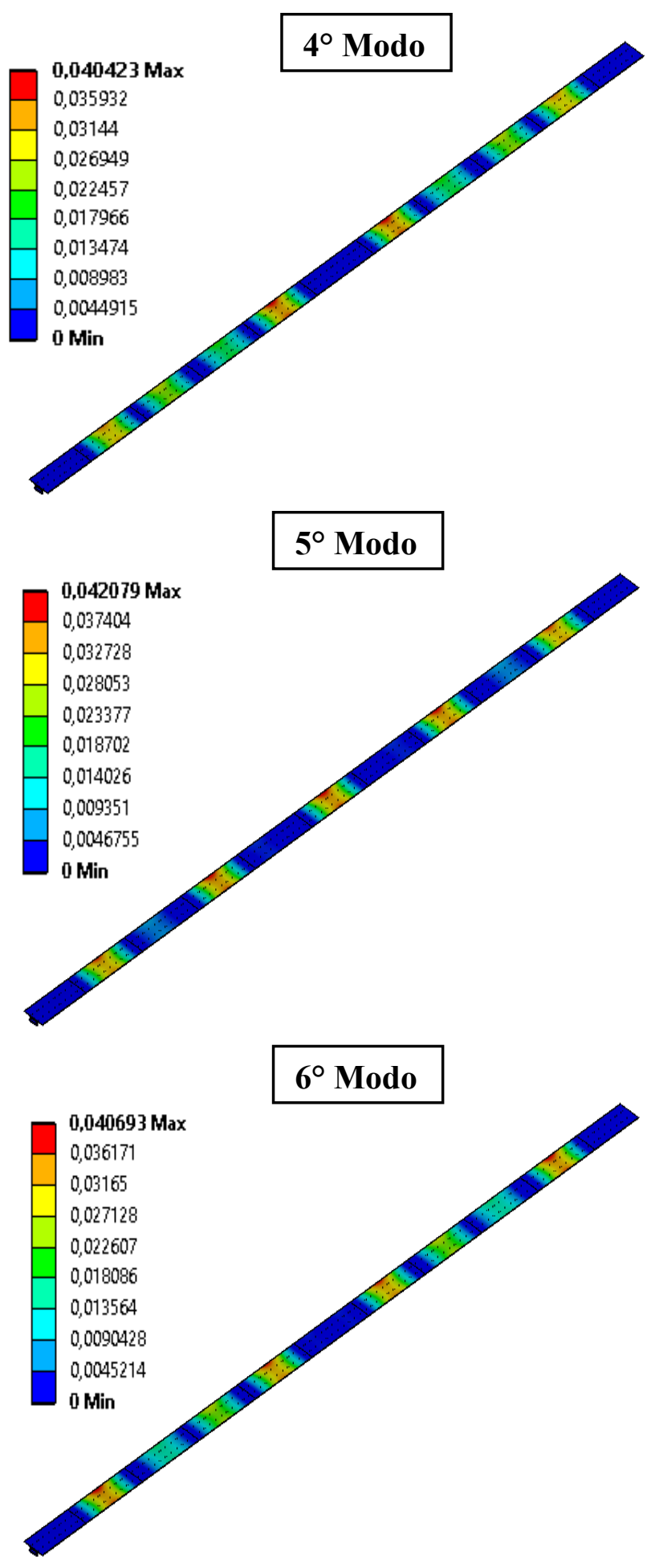

Figura 7. Deslocamentos totais para os 6 modos de vibração, unidade em milímetros. 
Observa-se que o primeiro modo, de frequência 3,09 Hz, apresentou um deslocamento máximo de $0,0413 \mathrm{~mm}$, sendo este deslocamento localizado no vão central da ponte, vão 6 , mais precisamente na borda lateral da laje no meio do vão. O segundo modo, de frequência $3,10 \mathrm{~Hz}$, apresentou 0,0407 mm de deslocamento máximo, localizado na borda lateral da laje no vão 4 .

O terceiro modo, de frequência $3,12 \mathrm{~Hz}$, apresentou um deslocamento máximo de $0,0422 \mathrm{~mm}$, sendo localizado também no vão central, como o primeiro modo. O quarto modo, de frequência $3,14 \mathrm{~Hz}$, apresentou deslocamento máximo de $0,0404 \mathrm{~mm}$, localizado na borda lateral da laje do vão 5 no meio do vão. O quinto modo, de frequência $3,17 \mathrm{~Hz}$, apresentou deslocamento máximo de $0,0421 \mathrm{~mm}$, localizado na borda lateral da laje, no meio do vão central. O sexto modo, de frequência $3,21 \mathrm{~Hz}$, com deslocamento máximo de 0,0407 localizado no meio do vão 10 , na borda lateral da laje.

Tabela 3. Frequência natural e deslocamento máximo.

\begin{tabular}{|c|c|c|}
\hline Modo & $\begin{array}{c}\text { Frequência } \\
(\mathbf{H z})\end{array}$ & $\begin{array}{c}\text { Deslocamento Máximo } \\
(\mathbf{m m})\end{array}$ \\
\hline 1 & 3,09 & 0,0413 \\
\hline 2 & 3,10 & 0,0407 \\
\hline 3 & 3,12 & 0,0422 \\
\hline 4 & 3,14 & 0,0404 \\
\hline 5 & 3,17 & 0,0421 \\
\hline 6 & 3,21 & 0,0407 \\
\hline
\end{tabular}

\section{CONCLUSÃO}

A análise dinâmica da estrutura via Método dos Elementos Finitos, possibilitou a identificação das frequências naturais, deslocamentos e participações de massa para 6 modos de vibração.

Os resultados mostraram que no $3^{\circ}$ modo de vibração, o deslocamento apresentou maior amplitude, com 0,0422 milímetros. $\mathrm{O}$ vão central da ponte foi a região que apresentou todos os máximos deslocamentos desta análise, resultando na região com a maior probabilidade de apresentar danos, pois contém maiores solicitações, sendo assim, a região mais vulnerável da estrutura. Vale ressaltar que é de suma relevância a primeira frequência obtida, pois a está frequência podem resultar em fenômenos oscilatórios danosos a estrutura da ponte.

Quanto as participações de massa, constatou-se as seguintes maiores participações para cada eixo, onde para o eixo X apresentou 50,26\% no quinto modo de vibração, para o eixo Y apresentou $81,29 \%$ no quinto modo de vibração, e para o eixo $Z$ apresentou $71,79 \%$ no sexto modo de vibração. Os resultados obtidos permitem uma maior compreensão a respeito das possíveis respostas da estrutura frente à esforços capazes de introduzir movimentos oscilatórios. Assim, identificou as regiões mais propicias a movimentações durante a ocorrência de uma excitação dinâmica na estrutura. Por fim, pode-se dizer que simulações dessa natureza possuem grande importância como ferramenta para identificação de mecanismos de danos. 


\section{REFERÊNCIAS BIBLIOGRÁFICAS}

Associação Brasileira De Normas Técnicas. (2003). NBR 8681: ações e segurança nas estruturas - procedimentos. Rio de Janeiro.

Pinheiro, L. M. (2007). Fundamentos do concreto e projeto de edifícios. Universidade de São Paulo, São Carlos. 